\title{
Outer limits of flow cytometry to quantify viruses in water
}

\author{
Elena Dlusskayaa ${ }^{a}$ Rafik Dey ${ }^{a, b}$, Peter C. Pollardc and Nicholas J. Ashbolta,b,d ${ }^{*}$ \\ a School of Public Health, University of Alberta, Edmonton, Canada \\ ${ }^{b}$ Dept. Medical Microbiology and Immunology, University of Alberta, Edmonton, Canada \\ c School of Engineering and the Built Environment, Griffith University, Queensland, Australia \\ ${ }^{d}$ School of Environment, Science and Engineering, Southern Cross University, Australia \\ * - corresponding author nick.ashbolt@scu.edu.au Telephone: +61 266203766
}

\section{Supporting Information summary:}

The supporting information consists of 2 figures (SI. 1 and SI. 2) 


\section{Supporting Information (SI):}

\section{SI. 1}
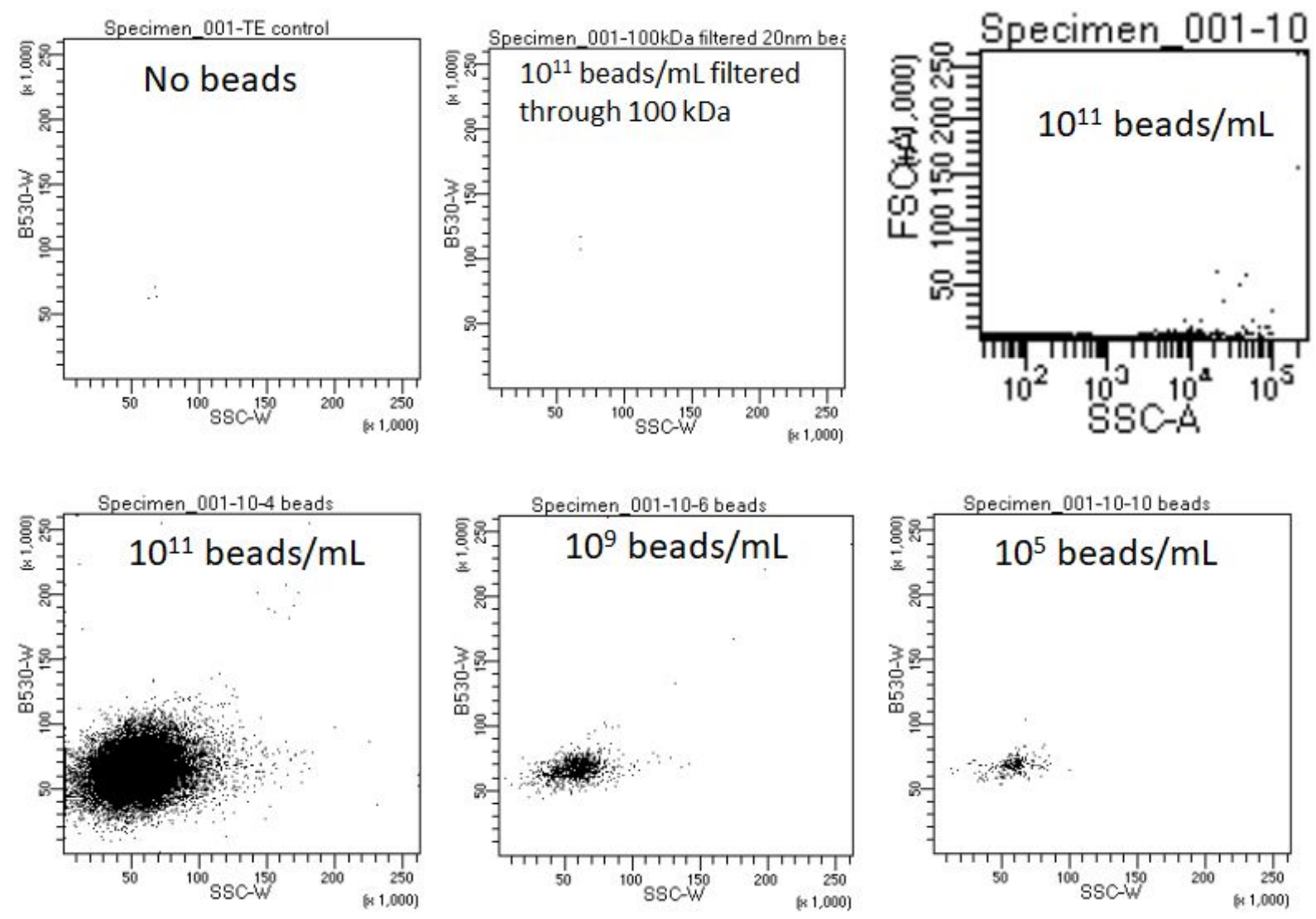

Fortessa X-20 flow cytometer was able to detect green-fluorescent beads $20 \mathrm{~nm}$ diameter (Thermofisher, Ref\# F8787) by fluorescence only and not by forward scatter at the voltage instrument settings we used for virus detection. However, only the brightest beads in the sample population were detected, as the total flow virometry counts were lower than the real bead concentration. 


\section{SI. 2}

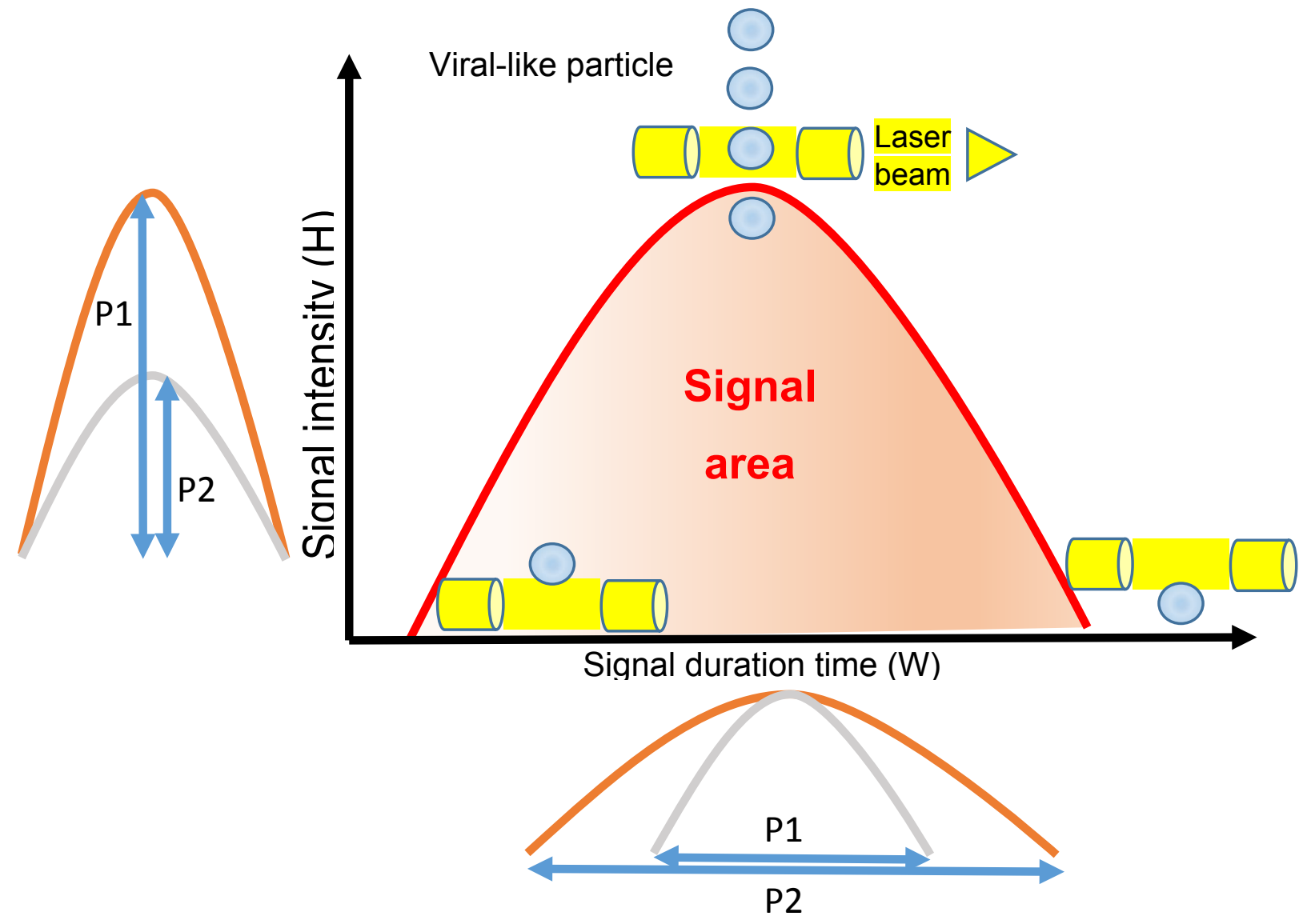

Sketch to explain flow cytometric signals used: The signal intensifies as a particle starts crossing the beam and reaches its maximum when the particle is in the middle. The signal then diminishes as the particle passes out of the beam. Populations (P1 and P2) of Viral Like Particles can be resolved based on the differences in signal duration (W) or signal intensity $(\mathrm{H})$. 